\title{
Representações sociais de pessoas vivendo com HIV: autopercepção da identidade ego- ecológica
}

\author{
The social representations of people living with HIV: self-perception \\ of the ego-ecological identity
}

Dayana Souza de Melo', Rosâne Mello²

DOI: 10.1590/0103-1104202113112

RESUMO Este artigo objetivou compreender a identidade ego-ecológica da pessoa vivendo com HIV e analisar as influências exercidas pelo profissional de saúde sobre esses indivíduos. A pesquisa é de abordagem qualitativa, com base teórico-metodológica na Teoria das Representações Sociais. Foram realizadas entrevistas com complementação de frases com 20 pessoas vivendo com HIV que recebem atendimento no laboratório de imunologia de um hospital universitário. O perfil populacional do estudo influenciou positivamente no lidar com o agravo, dada a maior idade e tempo de tratamento. Apesar disso, os estigmas sociais negativos ainda se fizeram presentes. Enfatizou-se o profissional de saúde com potencial de transformação, sendo este vinculado à oferta qualificada de cuidado. Por fim, concluiu-se que ainda há preconceitos e tabus direcionados a pessoas vivendo com HIV, indicando assim a necessidade de um olhar atencioso às vulnerabilidades biopsicossociais dessa população.

PALAVRAS-CHAVE HIV. Pessoal de saúde. Saúde mental. Estigma social.

ABSTRACT The aim of this paper is to understand the ego-ecological identity of the person living with $H I V$ and to analyze the influences exerted by the health professional on these individuals. The research has a qualitative approach, with theoretical and methodological basis on the Theory of Social Representations. Phrase-complementing interviews were conducted with 20 people living with HIV who receive care at the immunology laboratory of a university hospital. The population profile of the study had a positive influence on dealing with the problem, given the older age and treatment time; despite that, negative social stigmas were still present. The health professional with transformation potential was emphasized, being linked to the qualified offer of care. Finally, it was concluded that there are still prejudices and taboos directed at people living with HIV, thus indicating the need for a careful look at the biopsychosocial vulnerabilities of this population.

1 Universidade Federal do Rio de Janeiro (UFRJ), Instituto de Psiquiatria (Ipub) - Rio de Janeiro (RJ), Brasil.

dayanasouza_10@hotmail. com

2 Universidade Federal do Estado do Rio de Janeiro (Unirio), Escola de Enfermagem Alfredo Pinto (EEAP) - Rio de Janeiro

(RJ), Brasil.
KEYWORDS HIV. Health personnel. Mental health. Social stigma. 


\section{Introdução}

A situação de vulnerabilidade social de pessoas vivendo com HIV é assinalada em livros, filmes e demais exposições artísticas, produzidas, principalmente, nas décadas de 1980 a 1990. Apesar dessa ampla divulgação das necessidades individuais e dos dados epidemiológicos da síndrome, a Síndrome da Imunodeficiência Adquirida (Aids) ainda é cercada de estigmas e conflitos'.

A Aids é uma síndrome infecciosa crônica, causada pelo Vírus da Imunodeficiência Humana (HIV) e se caracteriza pela progressiva destruição do sistema imunológico, comprometendo especialmente a imunidade do tipo celular. Em vários casos, as infecções oportunas do déficit imunológico tornam-se letais ${ }^{2}$.

A síndrome se apresentou como pandemia em meados da década 1970, em um contexto estadunidense, com afecções de âmbito político, social e religioso. A Aids se tornou um estigma! Algo que não devia ser mencionado, que só dizia respeito a grupos minoritários e que tinham o que, considerável parte da população, chamavam de 'comportamento sexual promíscuo' (multiplicidade de parceiros e relações homoafetivas, entre outros)'

O problema atingiu níveis globais derivados, principalmente, do desconhecimento científico sobre o HIV, sendo mínimas as noções de diagnóstico, causa e tratamento. O que outrora era o mal das minorias sociais atingiu todas as classes, fazendo com que a criação de políticas públicas fosse essencial, inclusive no Brasil.

A descoberta de uma nova terapêutica farmacológica, chamada zidovudina (AZT), possibilitou aumentar o prognóstico e a qualidade de vida de quem portava tal vírus. Em 1991, o Sistema Único de Saúde brasileiro (SUS) passou a disponibilizar o AZT de forma gratuita. Assim, a Terapia Antirretroviral (ARV) mudou o cenário epidemiológico do País. Características marcantes da Aids, como emagrecimento e fragilidade física, foram se reduzindo ${ }^{3}$. Além disso, os impactos na morbimortalidade também se refletiram no número de internações e, consequentemente, na maneira como os profissionais de saúde e indivíduos se relacionam com as pessoas vivendo com HIV, pois estes vêm se mostrando cada vez mais ativos e inseridos socialmente.

Apesar dessa inserção progressiva bem-sucedida, estudar tal grupo social ainda se faz necessário. Comprovadamente, em pacientes que vivem com HIV, o risco de desenvolver depressão é o dobro do que na população geral, afetando sua qualidade de vida $\mathbf{4}^{\mathbf{4} 5}$. Além disso, temos a desinformação acerca do contágio e do tratamento farmacológico, e o medo da morte, que traz à tona uma situação de vulnerabilidade psicossocial. Nesse contexto, o domínio teórico faz-se primordial para o atendimento aos pacientes com tal comorbidade.

Para nortear a pesquisa, as seguintes questões foram trabalhadas: como o indivíduo vivendo com HIV se vê na sociedade? Como este acredita que os outros o veem? Qual é a imagem do profissional de saúde para as pessoas vivendo com HIV, bem como suas influências?

A pesquisa tem como objeto a autorrepresentação daqueles que vivem com HIV. Ou seja, conhecer a identidade, suas peculiaridades, por meio das representações que o indivíduo tem sobre si mesmo e o meio em que está inserido. Com isso, a Teoria das Representações Sociais desponta como referencial teórico-metodológico para compor tal estudo, pois permite o aprofundamento dessas questões.

Assim, Souza ${ }^{6(61)}$ discorre um pouco sobre a Teoria das Representações Sociais, dizendo que:

Representar é uma capacidade mental inerente ao homem que busca apreender a realidade que o rodeia. Pode-se perceber essa capacidade representativa através da linguagem falada e escrita, nos comportamentos, na produção pictórica e gráfica que nos cercam. Em síntese, pode-se afirmar que representar são formas que traduzem o querer, o sentir e o agir humanos.

O estudo objetiva, então, compreender a identidade ego-ecológica da pessoa vivendo 
com HIV e analisar as influências exercidas pelo profissional de saúde sobre esses indivíduos.

Compreender a identidade ego-ecológica diz sobre buscar um método mais sensível, que fundamenta as nossas práticas e que se propõe a analisar como o indivíduo se insere e se entende no mundo, problematizando, a partir daí, suas questões e conflitos. A Teoria Ego-ecológica, que foi pensada pela psicóloga social canadense Marisa Zavalloni, é uma das vertentes da Teoria das Representações Sociais, compondo o referencial teórico-metodológico deste trabalho? ${ }^{7}$.

\section{Material e métodos}

Trata-se de um estudo com abordagem qualitativa e exploratória. Por meio desta, objetivou-se o estreitamento da relação entre o ator social, inserido em seu contexto, e o pesquisador, visando sempre entender o processo, e não apenas o resultado ${ }^{8}$.

O estudo dividiu-se em três etapas: a) análise bibliográfica, para identificação da problemática; b) entrevistas com pessoas que tiveram experiências práticas com o problema em questão; e c) análise dos temas emergentes para obter a compreensão e alcançar os objetivos propostos?.

A coleta de dados deu-se por intermédio de entrevistas, com uma estrutura preexistente mais flexível a intervenções pontuais do entrevistador e colocações do respondente ${ }^{10}$. As perguntas são semiestruturadas, permitindo, assim, o surgimento de questões individuais do entrevistado"1.

Foram entrevistados 20 adultos em tratamento para HIV no laboratório de imunologia de um Hospital Universitário (HU), localizado no estado do Rio de Janeiro, sendo a coleta de dados realizada uma vez por semana, por um período de um mês.

A abordagem foi realizada no $\mathrm{HU}$, no qual foram explicitadas as finalidades do estudo, bem como solicitado o preenchimento do
Termo de Consentimento Livre e Esclarecido (TCLE). Em seguida, foi oferecida uma cópia com o Instrumento de Contextualização do Participante (ICP), contendo perguntas sobre condições socioeconômicas e acerca de sua história de saúde, no que diz respeito ao HIV.

Foi utilizado um inventário constituído por complementações de frases sobre as representações da identidade dos respondentes como pessoas vivendo com HIV. Destaca-se que tal inventário foi preenchido com uma palavra ou frases curtas. Este é baseado na Teoria Ego-ecológica, sendo uma das vertentes da Teoria das Representações Sociais, que permite conhecer a construção da identidade vislumbrada sob três esferas principais: as representações que o indivíduo tem de si mesmo, dos outros (ou seja, seu meio inserido mais próximo) e da sociedade 7 .

Assim, inicialmente, tomou-se como base a resposta do indivíduo a dois estímulos distintos: 'eu/nós' e 'eles'. Esse duplo estímulo (eu/nós e eles) propõe o aparecimento de um autopensamento crítico, bem como dos indivíduos que compõem sua matriz social7. A partir do estímulo 'eles', o entrevistado se afasta do grupo para que consiga opinar a tal respeito, enquanto o 'nós' traz reaproximação, com o reforço da ideia individualizada. Em seguida, foi solicitado que o entrevistado completasse as seguintes sentenças: 1.a) Eu, como pessoa vivendo com HIV, sou [...]; 1.b) Eles, como portadores de HIV, são [...].

Foi abordada, ainda, a visão que a sociedade e o profissional de saúde têm sobre a pessoa vivendo com HIV. Para tal, foi pedido que o entrevistado completasse as seguintes sentenças: 2.a) A sociedade vê o portador de HIV como [...]; 2.b) Os profissionais de saúde veem o portador de HIV como [...].

Após isso, o entrevistado foi convidado a confirmar as frases ditas por ele e se as afirmações são referentes à sua identidade (auto) ou a de outros (alo). Em um terceiro momento, o entrevistado verbalizou se os atributos trazidos por ele eram positivos ou negativos.

$\mathrm{Na}$ terceira etapa, buscou-se desmembrar 
suas afirmações, a fim de traçar os perfis subjetivos. Essa foi nomeada Contextualização Representacional, em que foram retomados termos e valores atribuídos à questão norteadora da pesquisa ${ }^{\mathbf{2}}$.

Logo, pretendeu-se correlacionar a história do indivíduo com as afirmações feitas na etapa anterior. Em tal momento, passou-se às seguintes formas analíticas: a) do perfil traçado sobre si mesmo; b) da construção de sua identidade, refletida nos seus grupos sociais mais próximos; c) dos valores atribuídos às definições coletivas e individuais?.

A análise deste estudo seguiu três momentos: 1) Entender: as respostas obtidas no estudo são lidas, a fim de retirar a ideia central de cada pensamento e tomar conhecimento dos achados subjetivos; 2) Sintetizar (análise temática): as informações foram divididas em categorias para criação de um roteiro de leitura de sequência lógica; 3 ) Criticar (análise interpretativa): inserção das ideias do analisador, sendo este corresponsável pela produção de todos os achados desde então ${ }^{13}$.

Em atendimento à Resolução CNS $n^{\circ}$ 466/2012, a pesquisa foi submetida ao Comitê de Ética em Pesquisa da Universidade Federal do Estado do Rio de Janeiro para apreciação, e somente após sua liberação, em 25 de junho de 2019, deu-se início à coleta de dados, sendo aprovada sob número de parecer 3.410.907.
Para a abordagem, o TCLE foi lido e assinado pelo participante, sem limite de tempo e com a entrevistadora disponível para esclarecimento de quaisquer dúvidas anteriores ou posteriores à realização do estudo. A entrevista foi realizada em sala reservada. Para garantir o sigilo dos entrevistados, os participantes foram identificados pela sigla EH (Entrevistado do Hospital), seguida por um código numérico individual. A pesquisa teve financiamento próprio e sem fins lucrativos. Acrescenta-se ainda que a pesquisa gerou um risco mínimo, dada a possibilidade de desconforto, por parte dos entrevistados, com o tema do estudo, por relembrar situações que vivenciaram.

\section{Resultados e discussão}

Participaram desta pesquisa 20 entrevistados, seguindo os critérios de inclusão e exclusão, com idade média de 50 anos. A maior parte do público foi do gênero feminino e, em sua totalidade, cisgênero. Em relação à orientação sexual, a maior incidência foi de indivíduos heterossexuais, seguidos de homossexuais. Quanto às questões específicas sobre o HIV, a forma de contágio mais relatada foi por via sexual. Todos os respondentes faziam tratamento exclusivamente farmacológico, cujo tempo médio era de 13 anos.

Quadro 1. Perfil dos entrevistados. Rio de Janeiro, 2019

\begin{tabular}{lll}
\hline Indicador & Intervalos & Porcentagem \\
\hline Idade & $20-29$ anos & $5 \%$ \\
& $30-39$ anos & $10 \%$ \\
& $40-49$ anos & $30 \%$ \\
& $50-59$ anos & $35 \%$ \\
\hline Gênero & $60-69$ anos & $20 \%$ \\
\hline Orientação Sexual & Feminino & $70 \%$ \\
& Masculino & $30 \%$ \\
\hline
\end{tabular}




\begin{tabular}{lll}
\hline Quadro 1. (cont.) & & \\
\hline Indicador & Intervalos & Porcentagem \\
\hline Escolaridade & Ensino fundamental & $35 \%$ \\
& Ensino médio & $50 \%$ \\
& Ensino técnico & $5 \%$ \\
& Ensino superior & $10 \%$ \\
\hline Forma de contágio & Relação sexual & $60 \%$ \\
& Transfusão Sanguínea & $10 \%$ \\
& Acidente de trabalho & $5 \%$ \\
& Desconhecido & $25 \%$ \\
\hline Tempo de tratamento para o HIV & $0-11$ meses & $10 \%$ \\
& $1-5$ anos & $10 \%$ \\
& $6-10$ anos & $15 \%$ \\
\hline
\end{tabular}

Ao estímulo ' $\mathrm{Eu}$, como portador de HIV, sou [...]', referente ao instrumento de representações da identidade ego-ecológica, obteve-se, em sua maior parte ( 37 respostas, $58,7 \%$ ), uma identificação positiva com o grupo. Ou seja, as complementações de frases indicaram satisfação e valorização, tanto de suas qualidades intrínsecas como de sua inserção no grupo de pessoas vivendo com HIV7. Com isso, é possível verificar que as pessoas vivendo HIV se sentem pertencentes ao meio e às suas relações, e com o agravo, não apresentam, em sua maior parte, um fator estressor.

Com o avanço do tratamento farmacológico e o aumento da qualidade de vida das pessoas vivendo com HIV, é perceptível o envelhecimento dessa população ${ }^{14}$. O perfil deste estudo comprova esse fato, marcado tanto pela média de idade quanto pelo tempo de tratamento elevados. Ambos os fatores auxiliam no sentimento de esperança e do menor impacto gerado pela doença como potencial fator estressor ${ }^{15}$. Dessa forma, a população com mais idade, e há mais tempo vivendo com HIV, lida melhor com questões relativas à doença se comparada à população mais jovem e com um diagnóstico recente.
Houve um número significativo de pessoas $(41,3 \%)$ que expressou ter identificação negativa com o grupo, ou seja, estas não se sentem contentadas, nem com o fato de viverem com HIV, nem com o seu meio social relativo ao agravo. Resta demonstrado que, apesar dos fatores capazes de auxiliar os indivíduos a lidarem com tais questões, como maior idade e maior tempo de tratamento para o HIV, ainda há um peso negativo envolvendo essa temática, gerando, por exemplo, sentimentos de vergonha.

A despeito da crescente produção de métodos de divulgação de conhecimentos específicos ao HIV, é percebida a falta de conhecimento sobre meios de contágio, prevenção e tratamento. Esses tabus dão origem a um distanciamento da sociedade para com o indivíduo que vive com HIV, estimulando a segregação e a exclusão em razão da desinformação ${ }^{16}$.

A segunda indagação, 'Eles, como portadores de HIV, são [...]', em sua maior parte, ou seja, em 24 respostas (42,1\%), não revelou identificação com o grupo. Essa diferenciação ocorre de forma negativa, ou seja, há uma desvalorização de questões pessoais ${ }^{7}$. O mesmo 
resultado pode ser visto na pergunta 'A sociedade vê o portador de HIV como [...]', na qual há diferenciação negativa com o grupo em 44 respostas (78,6\%). Quando há o distanciamento, por meio do estímulo 'eles', é possível identificar que a imagem negativa da pessoa vivendo com HIV ainda se faz presente e se perpetua no imaginário, inclusive dos próprios protagonistas desse cenário.

Estigma é definido como uma expressão da fuga do normal preestabelecido socialmente. Entre as circunstâncias descritas pelo autor para a produção desse fenômeno, o indivíduo que vive com HIV está atrelado não só a questões físicas, mas também a atributos éticos e morais de valores e caráter ${ }^{17}$.

Assim, "normais e estigmatizados são perspectivas que são geradas em situações sociais durante os contatos mistos, em virtude de normas não cumpridas que provavelmente atuam sobre o encontro", diz Goffman ${ }^{8(148)}$. No presente estudo, o desvio do padrão social desse grupo está relacionado com a expressão de gênero e sexualidade que está em conflito com o padrão social heteronormativo.

Destaca-se que 39 das complementações (70,9\%) quanto à questão 'Os profissionais de saúde veem a pessoa vivendo com HIV como [...]' apresentaram diferenciação positiva ao grupo, ou seja, mesmo não havendo identificação direta com profissionais de saúde, os participantes exaltam as qualidades destes, vendo seus diferenciais de modo favorável.

A mudança do perfil epidemiológico tornou o cuidado a tal agravo não restrito ao leito de uma enfermaria hospitalar. O cuidado e o aperfeiçoamento tiveram de ser adaptados para além do atendimento da internação. Com isso, a evolução de novas técnicas em dispositivos como Unidades Básicas de Saúde, ambulatórios e hospitais-dia fez-se necessária ${ }^{\mathbf{1 8}}$. Tanto essa iniciativa quanto a atualização corroboram os achados de tal estudo, visto que que os profissionais foram mencionados positivamente.

\section{Espaço elementar de identidade social: o não ser e o ser soropositivo}

Nesta etapa, a análise deu-se objetivando verificar o grau de identificação do sujeito com a complementação dita por ele ${ }^{7}$. Para isso, usou-se o conceito chamado Espaço Elementar de Identidade Social - um componente da Teoria Ego-ecológica que se destina a classificar se as afirmações foram, em sua maioria, egomórficas, isto é, atribuídas a si mesmo, ou alomórficas, isto é, atribuídas ao outro ${ }^{\mathbf{1 2}}$.

O presente estudo apresentou perfil egomórfico no estímulo 'eu/nós', e alomórfico no estímulo 'eles', sendo o primeiro majoritariamente positivo, e o segundo, em contrapartida, negativo. Houve tendência ao distanciamento do outro, sempre trazendo a imagem do soropositivo de forma negativa. Neste ponto, há de se considerar os preconceitos e os estigmas nas práticas e vivências dessa população. Quando existe essa aproximação, e as complementações mostram significados contrários, indica-se que os sujeitos não se sentem pertencentes a esse grupo, inferindo que viver com o HIV é algo com o qual não se quer estar associado.

Com base na análise temática, foram definidos os seguintes eixos: a) o ser normal e suas associações; b) expressão de sentimentos e emoções; c) relações com a doença; e d) demais implicações sociais relativas a viver com HIV. Sobre os eixos temáticos, a maior parte das citações, sendo 79 respostas (33,8\%), enquadrou-se no eixo das demais implicações sociais relativas a viver com o HIV. Em seguida, emergiu o eixo que diz respeito a ser normal e suas associações, com 66 respostas (28\%). Ambos trazem questões relativas a como se portar socialmente e discutem questões relevantes sobre estigmas, preconceitos e padrões socialmente aceitáveis.

Na subcategoria das identificações positivas com o grupo, foi mais mencionado o eixo 'expressão de sentimentos e emoções', com 22 complementações (46,8\%). Em identificações negativas com o grupo, o eixo mais mencionado foi o que aludia às demais implicações 
sociais diretas a pessoa que vive com o HIV, com 21 complementações (43,7\%). Na subcategoria das diferenciações positivas, a maioria foi 'o ser normal e suas associações', com 36 respostas $(58,0 \%)$. Por último, nas diferenciações negativas, deu-se 'demais implicações sociais diretas no portador de HIV', com 39 respostas (46,7\%), seguido do eixo 'relações com a doença', com 16 considerações $(20,8 \%)$.

\section{O ser normal e suas associações}

Foram identificados vocábulos como, "rotina" (EH2), "ser humano comum" (EH9) e "normal" (EH17), em contrapontos opostos como "diferente" (EH17), "anormal" (EH4) e "um ser alienígena" (EH18), sempre trazendo esse conceito da aproximação de um padrão que os difere ou não do restante da sociedade que não porta o HIV. Assim,

a sociedade estabelece os meios de categorizar as pessoas e o total de atributos considerados como comuns e naturais para os membros de cada uma dessas categorias ${ }^{\mathbf{1 9 ( 1 2 )}}$

Nesse eixo, há interesse em reforçar que o fato de conviver com HIV não compõe sua totalidade, ao mesmo tempo que os pacientes acusam a sociedade e alguns profissionais de saúde de não os verem de forma integral, somente por meio da doença. Aqui cabe destacar o conceito de integralidade do nosso SUS, que afirma que o sujeito deve ser visto de forma multiaxial, assim como é definido o conceito de saúde 20 .

\section{Expressão de sentimentos e emoções}

Foram identificados vocábulos como "feliz" (EH4), "forte" (EH9) e "perseverante" (EH20) como marcadores positivos. Já a contraparte negativa identifica sentir-se "culpado" (EH1), "revoltado" (EH4) e "triste" (EH16). Aqui, ser é o mesmo que sentir; as respostas foram mais rápidas e potencialmente interligadas com aquele dia em que ocorreu a entrevista. Além disso, aparecem questões muito subjetivas, bem como de foro íntimo.

Após as mais de três décadas do aparecimento da epidemia de Aids, é notável que as questões relativas ao HIV não estão apenas ligadas ao seu arcabouço biofisiológico. Tais associações permeiam os aspectos biopsicossociais, e observa-se um progressivo aumento de estudos que abordam questões íntimas da pessoa vivendo com HIV, dada a sua importância, que implica não só o tratamento, mas todo o meio em que está inserido²1.

\section{Relações com a doença}

Os entrevistados relatam questões conexas ao processo de adoecer com o HIV, entre as quais, estão a aceitação ou não da doença, os sintomas e os impactos em sua rotina e disposição física. Essas questões foram expressadas nas seguintes complementações: "ter consciência da doença" (EH4), "complicado de viver" (EH9) e "doente" (EH6). Sobre a forma de contágio, emergiram, com ênfase, as preocupações em contaminar o outro, trazendo a preocupação como algo positivo e a despreocupação como um ato de irresponsabilidade, marcado nas seguintes complementações: "procuro não contaminar ninguém" (EH13) e "descuidados por contaminar o outro" (EH3).

O conhecimento acerca da doença e seu manejo é visto positivamente e muito mencionado, principalmente relativo à sociedade e ao profissional de saúde. Possuir conhecimento técnico-científico e desenvolver ações de educação em saúde são métodos que podem influenciar na adesão ao tratamento, assim como na morbimortalidade e potencial qualidade de vida ${ }^{22}$. Em contraponto, as complementações "leprosos" (EH20) e "contagioso" (EH7, EH12) também foram citadas, reafirmando a necessidade de esclarecimentos e revelando o medo do contágio e o afastamento repentino daqueles que o cercavam. 


\section{Demais implicações sociais diretas a pessoa que vive com o HIV}

Foram identificados diversos tabus ao assunto, seguidos de esquiva ou fuga, evidenciados nas seguintes complementaçõ es: "fechados" (EH9) e "não tocam no assunto" (EH10). A esquiva do assunto se dá por medo de prejulgamentos ou vergonha de si e do outro. Além destas, foram relatadas frases associadas a discriminação, preconceito, exclusão, invisibilidade e até mesmo animalização, como "bicho" (EH11, EH18). Foi possível fazer uma averiguação de uma associação daquele que convive com HIV atrelado a comportamentos relativos à inconsequência, à promiscuidade, à maldade e à irresponsabilidade, reforçando arquétipos sociais negativos e levando a situações antiéticas, não profissionais ou imorais ${ }^{3}$.

Em contraponto, quando não há rejeição, discriminação, e existe cuidado e carinho, isso é encarado de forma positiva, mostrando, assim, que ser e se sentir acolhido pelos outros é importante. O cuidado sem preconceito é valorizado, bem como reconhecido como potencial para resolver suas questões pessoais, como autoestima. A complementação "carinho" (EH1, EH12 e EH13) é citada várias vezes, principalmente vinda dos profissionais de saúde, sendo estes os mencionados: enfermeiros, técnicos de enfermagem, médicos e psicólogos. Resta explicitada a importância da criação de vínculo e do relacionamento interpessoal.

\section{Considerações finais}

A população mais velha e com um tempo maior de tratamento tende a lidar melhor com o fator
HIV. Apesar disso, a estigmatização é notável nas falas, principalmente no que concerne aos padrões aceitáveis, aos conceitos de normalidades e negativa associação com promiscuidade e imoralidade: estes ainda permanecem fortemente associados.

O conhecimento sobre questões específicas do HIV tem potencial de transformação. No caso de sua ausência, gera desconfortos diversos, por causa da desinformação sobre contágio e prevenção, o que provoca sentimentos de exclusão e vergonha. Em contrapartida, quando há esse conhecimento, transmite-se esperança e fortalecimento dos vínculos.

Os profissionais de saúde, imersos nesse ambiente, são vistos de forma positiva nas falas dos entrevistados. Os cuidados humanizados e éticos ultrapassam os conhecimentos técnico-científicos e são abordados como potencial para melhoria da qualidade de vida de pessoas com HIV.

Assim, tal trabalho alcançou seus objetivos dispostos, contribuindo para um maior entendimento de como se dá a identidade ego/social do perfil estudado. Seu resultado indica a necessidade da ampliação do olhar para além das questões fisiológicas do agravo, entendendo que as pessoas vivendo com HIV têm questões específicas que impactam seu modo de se relacionar com o mundo, suas construções de vínculos e sua qualidade de vida.

\section{Colaboradoras}

Melo DS (0000-0003-1464-829X)* e Mello R (0000-0001-6042-4647)* contribuíram igualmente para a elaboração do manuscrito. 


\section{Referências}

1. Zolin KP, Oliveira SF, Mendonça MK, et al. Os estigmas e a discriminação social e os portadores do vírus HIV a partir do filme "The normal heart”. [acesso em 2019 jan 10]. Disponível em: http://conferencia2016. redeunida.org.br/ocs/index.php/congresso/2016/paper/view/5050.

2. Brasil. Ministério da Saúde, Secretaria de Vigilância em Saúde. Cuidado integral às pessoas que vivem com HIV pela atenção básica: manual para a equipe multiprofissional. Brasília, DF: Ministério da Saúde; 2015.

3. Costa MS. A concepção das pessoas com HIV/AIDS acerca do estigma e da discriminação: um estudo junto ao serviço de assistência especializada em HIV/AIDS e Hepatites virais (SAE) do município de Campina Grande-PB. [monografia]. Campina Grande: Centro de Ciências Sociais Aplicadas, Universidade Estadual da Paraíba; 2015. [acesso em 2019 jan 10]. Disponível em: http://dspace.bc.uepb.edu.br/jspui/handle/123456789/9422.

4. São Paulo. Secretaria de Estado da Saúde, Coordenadoria de Controle de Doenças. Diretrizes para implementação da rede de cuidados em IST/HIV/AIDS: manual de assistência. São Paulo: SESSP; 2017.

5. Santos AP, Braide ASG, Silva PGB, et al. Fatores associados à qualidade de vida de pessoas com HIV/ AIDS. Cadernos ESP/CE. 2019 [acesso em 2019 ago 12]; 13(1):27-36. Disponível em: http://cadernos.esp. ce.gov.br/index.php/cadernos/article/view/166/159.

6. Souza DB. Representações sociais sobre indisciplina em sala de aula dos professores iniciantes da rede municipal de Presidente Prudente-SP: implicações para a formação inicial. [dissertação]. Presidente Prudente: Universidade Estadual Paulista; 2005.

7. Mello R, Furegato ARF. Teoria Ego-ecológica e o estudo da identidade social- aplicabilidade em pesquisas de enfermagem. Esc Anna Nery (impr.). 2011 [acesso em 2019 mar 30]; 15(4):825-832. Disponível em: http://dx.doi.org/10.1590/S1414-81452011000400023.
8. Bauer MW, Gaskell G. Pesquisa qualitativa com texto, imagem e som: um manual prático. Petrópolis: Vozes; 2017.

9. Gil AC. Métodos e técnicas de pesquisa social. 7. ed. São Paulo: Atlas; 2019.

10. Batista EC, Matos LAL, Nascimento AB. A entrevista como técnica de investigação na pesquisa qualitativa. Rev Interdiscip Cient Ap. 2017 [acesso em 2019 mar 30]; 11(3):23-38. Disponível em: https://rica.unibes. com.br/rica/issue/view/63/showToc.

11. Alonso A, Lima M, Almeida R. Métodos de pesquisa em Ciências Sociais: bloco qualitativo. São Paulo: Sesc São Paulo; CEBRAP; 2016.

12. Zavaloni M, Louis-Guérin C. Identité sociale et conscience. Introduction à l'égo-écologie. Montreal: Les Presses de L’Université de Montreal; 1984.

13. Rampazzo L. Metodologia Científica para alunos dos cursos de graduação e pós-graduação. 3. ed. São Paulo: Loyola; 2005.

14. Affeldt AB, Silveira MF, Barcelos RS. Perfil de pessoas idosas vivendo com HIV/AIDS em Pelotas, sul do Brasil, 1998 a 2013. Epidemiol Serv Saúde. 2015 [acesso em 2019 ago 5]; 24(1):79-86. Disponível em: http://dx.doi.org/10.5123/S1679-49742015000100009.

15. Cassétte JB, Silva LC, Felício EEAA, et al. HIV/AIDS em idosos: estigmas, trabalho e formação em saúde. Rev Bras Geriatr Gerontol. 2016 [acesso em 2019 ago 5]; 19(5):733-744. Disponível em: https://doi. org/10.1590/1809-98232016019.150123.

16. Andrade RG, Iriart JAB. Estigma e discriminação: experiências de mulheres HIV positivo nos bairros populares de Maputo, Moçambique. Cad. Saúde Pública. 2015 [acesso em 2019 set 10]; 31(3):565-574. Disponível em: https://doi.org/10.1590/0102-311X00019214.

17. Gunther LE. O HIV e a AIDS: preconceito, discriminação estigma no trabalho: aplicação da Súmu- 
la 443 do Tribunal Superior do Trabalho. Rev Eletr do TRT9. 2015 [acesso em 2019 set 10]; 42(4):46-67. Disponível em: https://juslaboris.tst.jus.br/handle/20.500.12178/90992.

18. Goffman E. Estigma: notas sobre a manipulação da identidade deteriorada. Rio de Janeiro: LTC; 1975.

19. Zambenedetti G, Silva RAN. Descentralização da atenção em HIV-Aids para a atenção básica: tensões e potencialidades. Physis Rio J. 2016 [acesso em 2019 set 10]; 26(3):785-806. Disponível em: https://doi. org/10.1590/S0103-73312016000300005.

20. Kalichman AO, Ayres JRCM. Integralidade e tecnologias de atenção à saúde: uma narrativa sobre contribuições conceituais à construção do princípio da integralidade no SUS. Cad. Saúde Pública. 2016 [acesso em 2019 set 10]; 32(8):e00183415. Disponível em: https://doi.org/10.1590/0102-311X00183415.
21. Villela WV, Monteiro S. Gênero, estigma e saúde: reflexões a partir da prostituição, do aborto e do HIV/AIDS entre mulheres. Epidemiol Serv Saúde. 2015 [acesso em 2019 set 10]; 24(3):531-540. Disponível em: https://www.scielosp.org/article/ress/2015.v24n3/531$540 /$.

22. Coutinho AFP, Lopes JL, Carneiro CS, et al. Satisfação dos pacientes atendidos no Ambulatório de Educação em Saúde. Rev Fund Care Online. 2019 [acesso em 2019 set 10]; 11(5):1111-1116. Disponível em: http:// dx.doi.org/10.9789/2175-5361.2019.v1li5.1111-1116.

Recebido em 01/02/2021

Aprovado em 10/09/2021

Conflito de interesses: inexistente

Suporte financeiro: não houve 